\title{
Selection of basal medium for culturing human umbilical cord mesenchymal stem cells in combination with human platelet lysate
}

\section{Lingjuan Wang}

Tian Qing Stem Cell Co., Ltd.

Chunxiang Liu

Tian Qing Stem Cell Co., Itd.

Huiying Lu

Tian Qing Stem Cell Co., Ltd.

JUN YAN ( $\sim$ junyan1@yahoo.com)

TIAN QING Stem Cell Inc. https://orcid.org/0000-0002-2886-3938

\section{Yi Zhang}

Tian Qing Stem Cell Co., Ltd.

\section{Research}

Keywords: Cell culture, Culture medium, Platelet lysate, Umbilical cord mesenchymal stem cells

Posted Date: July 16th, 2020

DOl: https://doi.org/10.21203/rs.3.rs-37421/v1

License: (9) This work is licensed under a Creative Commons Attribution 4.0 International License. Read Full License 


\section{Abstract \\ Background}

Different basal media have very different effects on cell growth, proliferation and differentiation potential of cultured human umbilical cord mesenchymal stem cell (hUC-MSC). This issue has not gotten enough research. The goal of this study is to select high safety and low cost basal media for hUC-MSC, which performs best in combination with commercial human platelet lysate product for cell proliferation and differentiation functions, especially suitable for high-passage hUC-MSC cultures.

\section{Methods}

Three different basal media were combined with 5\% UltraGROGAdvanced to culture P0 to P8 passage hUC-MSC. Cell morphology, cell expansion ratio, proliferation, differentiation and cell surface markers were examined and analyzed in order to draw conclusions.

\section{Results}

When observing the same passage under different culture systems, the number of cells obtained in MSCBM and a-MEM were higher than that cultured in IMDM $(0.01<P<0.05)$. The results between MSCBM and a-MEM were not significantly different $(P>0.05)$. Combining growth curve measurement and cell doubling time calculation, it was found that the cell proliferation functions of the three culture media were ranked as MSCBM, a-MEM and IMDM from high to low. The P8 cells cultured in different media all yield good clonal formation, and also all differentiated well. The number of cells differentiated into osteoblasts and chondrocytes cultured in different media was not significantly different $(P>0.05)$, while the number of adipocyte differentiation in MSCBM and a-MEM cultures were significantly higher than that of IMDM culture $(0.01<P<0.05)$. The cells in all cultures uniformly express high levels of MSC surface markers CD73, CD90, CD105, and negatively express CD34, CD45.

\section{Conclusions}

Taking all indicators into consideration, we conclude that the culture system of MSMBM supplemented with $5 \%$ UltraGROGAdvanced is the best for culturing hUC-MSC witha-MEM follows right after. This study revealed the importance of basal media in MSC cultures, and offer guidance on their selective use.

\section{Background}

Mesenchymal stem cells (MSCs) are a group of multipotent stem cells with the properties for self-renewal and multi-directional differentiation. When expanded in vitro, they are able to differentiate into many kinds of cells including chondrocytes, adipocytes, osteoblasts, nerve cells and muscles. MSCs generally 
positively express CD105, CD90, CD73, but do not express CD34, CD45 and other hematopoietic stem cell (HSC) markers. A large number of clinical trials are in progress, and several clinical studies have shown that MSCs plays a good role in the treatments of various diseases. Studies have shown that injection of MSCs can increase the molecular levels of IL-10, IL-37 and IL-17, and has better effects in improving ischemic myocardium [16]. MSCs have good therapeutic effects on nervous system injuries [1-3], musculoskeletal injuries [4], liver and lung diseases and dysfunctions [5], liver and lung fibrosis [6], immune system diseases, etc. MSCs also participate in the body's immune regulation and cell growth. All these results bring new hope for the treatment of many difficult diseases.

MSCs come from a wide range of sources. They were first discovered in human bone marrow. Later, researchers successively isolated and them from fat, umbilical cord, placenta and other tissues. Umbilical cord as a perinatal source for MSCs, are not prone to viral infections under the maternal placental barrier protection. HUC-MSC offers the advantages of abundant sources, simple isolation and culture, strong in vitro proliferation, in vivo properties including paracrine and other functions, low immunogenicity, very low HLA-\surface antigen expression, and no ethical disputes. Thus it has become the main source of MSCs for clinical research.

Many MSC preparations have been used in clinical research. MSCs can be cultured in vitro on large scales. Traditionally, MSCs have been cultured on a large scale with media supplemented with animalderived fetal bovine serum (FBS). In recent years, studies have shown that allogeneic platelet lysates are superior to FBS in culturing MSCs [5, 6, and 7]. MSCs cultured with platelet lysates have strong differentiation abilities and can maintain good cell morphological structures [8, 9]. It was found that the immune functions of cells cultured in platelet lysate was lower than that of cells cultured in FBS $[10,11]$. This was supported with a report of 13 patient's treatment with allogeneic MSCs expanded using medium containing platelet lysate [12]. Recent data on the use of MSCs to treat GVHD showed that $77 \%$ of medical institutions use platelet lysate-containing media to expand MSCs ( $54 \%$ comes from the blood bank, $23 \%$ comes from commercial sources), while the rest $23 \%$ of the institutions still using FBS [13]. When bone marrow and fat-derived MSCs were cultured with media containing FBS, human serum ,or human platelet lysate,the report found that fat-derived MSCs cultured with platelet lysate have better initial growth, but lower later proliferative functions than those using FBS and human serum $\square$ while bone marrow-derived MSCs grow best in platelet lysate culture system $[14,15]$. These studies did not distinguish the types of basal media used, so how basal media impact the cultivation of MSCs was unknown!

At present, the methods for cultivating hUC-MSC are by no means perfect. There are many kinds of basal media on the market. The formulation and ingredient quantities of each medium are different. There are differences in cell functions, cell recovery, cell purity, etc. So they are not all best suitable for hUC-MSC culture. The purpose of this study was to select a basal medium with good stability and low cost, when combined with animal-free platelet lysate, can ensure the high-passage hUC-MSC cultures of stability, proliferation, differentiation, immune-phenotype expression, and maintaining the MSC characteristics. 
In this paper, umbilical cord tissue block adherence-migration plating method was used to isolate MSCs. Three commonly used basal media with high performance and low cost were used for comparison. HUMMSCs were cultured with MSCBM from Dakewe, a-MEM and IMDM from Hyclone, combined with platelet lysate product UltraGROGAdvanced respectively. Functional tests on cells of different passages were performed. Basal medium which is best suited for Low-cost large-scale cultivation of hUN-MSCs was chosen based on the indicators of cell morphology, growth cycle, growth status, cell number, cell proliferation function, cell surface phenotype expression, cell differentiation function and others.

\section{Materials And Methods}

\section{Equipments and reagents}

CKX31 and IX51 inverted phase contrast microscope (OLYMPUS, Japan); flow cytometer (BD company, US); biological safety cabinet (Su Jing An Tai, China); CO2 incubators (Thermo, US).100uM cell Strainers (BD company, US); T75 cell culture flasks, 96-well culture plates and centrifuge tubes (Corning company, US); MSCBM (Mesenchymal Stem Cell Basal Medium, Dakewe, China); a-MEM and IMDM (Hyclone, US); UltraGROGAdvanced (Helios BioScience,US); 0.25\% TrypLE (Life Sciences, US); PE or FITC labeled CD105, CD34, CD45, CD90, CD73 antibodies (eBioscience『US).

\section{Human Umbilical Cord Mesenchymal Stem Cell Culture Methods}

In a biological safety cabinet, the umbilical cord is disinfected with $75 \%$ alcohol briefly, and then washed twice with physiological saline. In a dish, the cleaned umbilical cord was cut into sections of $2-3 \mathrm{~cm}$ in length, and the Wharton's jelly was peeled off. The Wharton's jelly was transferred into a centrifuge tube and was cut into 1-2 $\mathrm{mm}^{3}$ blocks using surgical scissors. The tissue blocks were plated at 2 grams tissue per T75 bottle, cultured with different basal media plus 5\% UltraGROGAdvanced, placed in a carbon dioxide incubator and incubate at $37{ }^{\circ} \mathrm{C}$ with $5 \% \mathrm{CO} 2$. The media were change after 4-5 days. When reached $70 \%$ confluent or more, the cells were digested with $0.125 \%$ TrypLE to obtain single cells, and plated at $1 \times 10^{6}$ cells / T175 flask, cultured with the same medium as before. After the cells had reached $90 \%$ confluent, the cells were passaged again. The cells are passaged and cultured up to the 8 th passage (P8) according to this method.

\section{Immunoassay For Cellular Phenotypes}

P1, P3, P5, P8 hUC-MSCs were digested into single cells with $0.125 \%$ TrypLE, and collected by centrifugation. After washing twice with physiological saline and cell counting, $1 \times 10^{6}$ cells were incubated with CD105, CD34, CD45, CD73, and CD90 antibodies at $4{ }^{\circ} \mathrm{C}$ in the dark for more than 30 
minutes. Washed three times with PBS, and the expression of immune-phenotypes were detected with flow cytometry, and a negative control was also set.

\section{Detection Of Cell Proliferation Function In Different Passages}

P1, P3, P5, P8 hUC-MSCs were digested into single cells with $0.125 \%$ TrypLE, and centrifuged to collect the cells. After washing twice with saline and cell counting, adjust the cell concentration to $1000 \mathrm{cells} / \mathrm{ml}$ and plate into 24-well plates at $0.5 \mathrm{ml} /$ well and cultured again. 24hrs later, three wells from each condition were digested and counted. This was done at the same time every day for 8 days. Each passage of cells was subjected to the same methods of growth curve drawing and cell proliferation function detection. The time of cell multiplication and doubling in different culture systems were calculate with the formulation DT = maximum number of doubling days $\mathrm{x}$ [lg2 / (lg maximum cell number-lg minimum cell number)] x24.

\section{Detection Of Cell Differentiation Function}

Osteogenic differentiation: P8 hUC-MSCs were digested into single cells with $0.125 \%$ TrypLE, and collect the cells by centrifugation. After washing twice with saline and cell counting, adjust the cell concentration in osteogenic differentiation medium to $1 \times 10^{4} \mathrm{cells} / \mathrm{ml}$ and plate into 24 -well plates with cover slips at 1 $\times 10^{4}$ cells/well. Setting up three parallel wells for each condition also set up one negative control well. The medium was changed the next day, and every 3 days afterward. On the 14th day in culture, the cells were rinsed gently with PBS once, fixed for 30 minutes in $4 \%$ paraformaldehyde, stain with Alizarin $S$ for 5 minutes, washed once, stained nuclei with hematoxylin for 1 minute, washed with water for 1 minute, dehydrated with gradient alcohol for two minutes, clarified with Xylene for 5 minutes, and mounted with glycerin gelatin. Observed under the microscope and randomly selected 5 fields to count positively stained cells for statistical analysis.

Adipogenic differentiation: The analysis was done in the same manner as stated above for osteogenic differentiation except the use of adipogenic differentiation medium and the staining with Oil Red 0 for 15 minutes instead.

Chondrogenic differentiation: The analysis was done in the same manner as stated above for osteogenic differentiation except the use of cartilage differentiation medium and the staining with Toluidine blue for 30 min instead.

\section{Observation Of Clone Formation Rate}


Digest the P8 hUC-MSCs into single cells with $0.125 \%$ TrypLE, and collect the cells by centrifugation. After washing twice with saline and cell counting, plated 600 cells in three $10 \mathrm{~cm}$ cell culture dishes with $8 \mathrm{ml}$ of medium per dish. The medium was change every 3 days. After culturing for 14 days, the cells were fixed for 30 minutes, stained with gentian violet, and colony formation was observed under a microscope.

\section{Cell Morphology Observation}

Observe and record the cell expansion and cell morphology of different experimental groups every day under an inverted phase-contrast microscope.

\section{Statistical analysis}

All data was analyzed and processed with SPSS17.0 statistical software. The results were expressed as Mean \pm SD. The comparison among multiple groups was done with one-factor analysis of variance, and the comparison between two groups was done with Student t-test.

\section{Results}

In this study, Wharton's jelly was isolated from selected freshly collected umbilical cord, shredded and adhered to culture flasks. MSCBM, a-MEM and IMDM medium containing 5\% platelet lysate were used for culture respectively. The medium was changed every 5 days. Up to 8 passage expansion were carried out. The morphology, growth cycle, proliferation function, doubling time, differentiation function, and cell surface markers of different passages were studied.

\section{Cell Morphology Observation And Proliferation Effects}

First, cell morphology observation revealed that there were no significant differences in the morphology of P0 to P5 passage cells cultured in all three different basal media $(P>0.05)$. The difference in morphology was more significant after P5, showing that the MSCBM and a-MEM cultured cells had uniform morphology and sizes, radial, and obvious three-dimensional looking, On the other hand,IMDM cultured cells had larger areas than MSCBM anda-MEM cultured cells $\square$ and the cells are flat and uneven in shapes $\square$ had rather poor three-dimensional looking,. The measurement results under microscope show that, from P6 to P8 passage, the volumes of IMDM cultured cells are larger than that of MSCBM and aMEM cultured cells $(0.01<\mathrm{P}<0.05)$, while the MSCBM and a-MEM cultured cells showed no significant difference when compared with P0 to P5 cultures $(P>0.05)$ (Fig. 1).

By observing the same passage cell proliferation in different culture systems (Table 1), it was found that the numbers of cells obtained by MSCBM and a-MEM culture were higher than that of the IMDM $(0.01<\mathrm{P}$ $<0.05)$, but there was no significant different between the first two kinds of media $(P>0.05)$. The doubling time of P3, P5, and P8 passage cells (Table 2) indicated that it was significantly longer in IMDM than in 
MSCBM and a-MEM $(0.01<\mathrm{P}<0.05)$. There was no significant difference in cell doubling time between the latter two $(P>0.05)$. The combination of growth curve determination and cell doubling time calculation revealed that the cell proliferation functions of the three culture media are ranked as MSCBM, a-MEM, and IMDM from high to low. The cell proliferation function of MSCBM culture was significantly higher than that of IMDM $(0.01<P<0.05)$, but was not significantly different from a-MEM $(P>0.05)$.

The clonal formation rate and the size of the formed clonal cell mass also reflect the cell proliferation functions (Table 3). The number of clones formed by P8 cells cultured in different media are different (the cell cluster with more than 15 cells was counted as one cloned cell cluster). After 60 hours in culture, the clonal cluster numbers formed by the cells in different media, and the cell numbers in each clonal cluster $\square$ are different. The clonal cluster numbers and the number of cells in each clone cluster formed by MSCBM cultured cells are higher than those formed by cells cultured in either a-MEM or IMDM $(0.01<P<$ 0.05).

Figure 1. The morphological observations and growth curves.

Photographs of phase-contrast $(a, b, c)$ and gentian violet staining $(d, e, f)$ of P8 passage cells cultured in MSCBM, IMDM, and a-MEM. $g$, h, and i are the growth curves of P3, P5, and P8 cells cultured in IMDM, MSCBM, and a-MEM, respectively.

Table 1

The number of human umbilical cord mesenchymal stem cells expanded at different passages $\left(X 10^{6} /\right.$ T75)

\begin{tabular}{|llllllllll|}
\hline & P0 & P1 & P2 & P3 & P4 & P5 & P6 & P7 & P8 \\
\hline a-MEM & $0.48 \pm$ & $2.85 \pm$ & $3.87 \pm$ & $3.98 \pm$ & $3.95 \pm$ & $3.89 \pm$ & $3.59 \pm$ & $3.55 \pm$ & $3.60 \pm$ \\
& 0.05 & 0.15 & 0.19 & 0.21 & 0.29 & 0.31 & 0.33 & 0.34 & 0.20 \\
IMDM & $0.48 \pm$ & $2.80 \pm$ & $3.50 \pm$ & $3.82 \pm$ & $3.70 \pm$ & $3.51 \pm$ & $2.86 \pm$ & $2.60 \pm$ & $2.85 \pm$ \\
& 0.02 & 0.17 & 0.16 & 0.31 & 0.31 & 0.26 & 0.27 & 0.21 & 0.23 \\
MSCBM & $0.70 \pm$ & $3.76 \pm$ & $4.47 \pm$ & $4.82 \pm$ & $4.88 \pm$ & $4.83 \pm$ & $4.87 \pm$ & $4.85 \pm$ & $4.87 \pm$ \\
& 0.01 & 0.19 & 0.17 & 0.25 & 0.38 & 0.27 & 0.31 & 0.30 & 0.24 \\
\hline
\end{tabular}

Table 2

The doubling time of umbilical cord mesenchymal stem cells of different passages (h)

\begin{tabular}{|llll|}
\hline \multicolumn{1}{|l}{ P3 } & P5 & P8 \\
\hline a-MEM & $21.8 \pm 2.5$ & $22.2 .3 \pm 2.3$ & $35.8 \pm 3.8$ \\
\hline IMDM & $28.5 \pm 3.3$ & $26.8 \pm 2.2$ & $41.6 \pm 4.1$ \\
\hline MSCBM & $20.2 \pm 2.3$ & $21.1 \pm 1.8$ & $33.5 \pm 4.2$ \\
\hline
\end{tabular}


Table 3

Umbilical cord mesenchymal stem cell clone formation data (clones / $10 \mathrm{~cm}$ dish and cell number/clone)

\begin{tabular}{|lll|}
\hline & clones / $10 \mathrm{~cm}$ dish & cell number/clone \\
\hline a-MEM & $19.6 \pm 0.4$ & $17.6 \pm 1.1$ \\
\hline IMDM & $17.5 \pm 0.6$ & $15.6 \pm 1.3$ \\
\hline MSCBM & $23.5 \pm 0.6$ & $21.5 \pm 0.9$ \\
\hline
\end{tabular}

\section{Comparison Of Cell Differentiation Functions}

Cells cultured in three different media all have strong differentiation functions. Counting differentiated cells under microscope revealed that the number of cells differentiated into osteoblasts and chondrocytes by $P 8$ cells cultured in different media was not significantly different $(P>0.05)$. However, the cell numbers of adipocyte differentiation by MSCBM and a-MEM cultured P8 cells were significantly higher than that of the same-passage cells cultured with IMDM $(0.01<\mathrm{P}<0.05)$ (Fig. 2$)$.

Figure 2 Osteogenic, adipogenic and chondrogenic differentiation by P8 hUC-MSCs.

$a, b$, and $c$ are Alizarin S staining for osteogenic differentiation of cells cultured in IMDM, MSCBM, and aMEM M, respectively, $d$ is a negative control; $e, f$, and $g$ are Oil Red staining for adipogenic differentiation of IMDM, MSCBM, and a-MEM cultured cells, respectively, $\mathrm{H}$ is a negative control; $\mathrm{i}, \mathrm{j}$, and $\mathrm{k}$ are Toluidine blue staining for chondrogenic differentiation of IMDM, MSCBM, and a-MEM cultured cells, I is a negative control.

\section{Comparison of cell surface marker detection}

The detection of cell surface markers by flow-cytometry showed that cells of different passages cultured in IMDM, MSCBM and a-MEM all positively expressed CD73, CD90 and CD105 at ratios over 99\%. Meanwhile, the expressions of CD34 and CD45 were both negative $(<2 \%)$. The difference between different media was not significant $(P>0.05)$ (Table 4). 
Table 4

Expression ratios of cell surface markers by different passage cells cultured in IMDM, MSCBM, and a-MEM.

\begin{tabular}{|lllllll|}
\hline Passage & Medium & CD34 & CD45 & CD73 & CD90 & CD105 \\
\hline P1 & a-MEM & $0.58 \pm 0.06$ & $0.29 \pm 0.08$ & $99.43 \pm 0.02$ & $99.92 \pm 0.03$ & $99.71 \pm 0.02$ \\
\cline { 2 - 7 } & IMDM & $0.49 \pm 0.09$ & $0.42 \pm 0.01$ & $99.71 \pm 0.05$ & $99.98 \pm 0.06$ & $99.86 \pm 0.04$ \\
\cline { 2 - 7 } P3 & MSCBM & $0.83 \pm 0.05$ & $0.78 \pm 0.01$ & $99.56 \pm 0.01$ & $99.97 \pm 0.01$ & $99.85 \pm 0.02$ \\
& a-MEM & $0.49 \pm 0.06$ & $0.79 \pm 0.05$ & $99.73 \pm 0.04$ & $99.93 \pm 0.02$ & $99.87 \pm 0.01$ \\
\cline { 2 - 7 } & IMDM & $1.05 \pm 0.04$ & $0.97 \pm 0.02$ & $99.87 \pm 0.01$ & $99.98 \pm 0.01$ & $99.96 \pm 0.02$ \\
P5 & MSCBM & $0.36 \pm 0.02$ & $0.19 \pm 0.02$ & $99.95 \pm 0.02$ & $99.99 \pm 0.03$ & $99.86 \pm 0.01$ \\
& a-MEM & $1.18 \pm 0.03$ & $0.81 \pm 0.02$ & $99.98 \pm 0.02$ & $99.99 \pm 0.02$ & $99.95 \pm 0.02$ \\
& IMDM & $1.23 \pm 0.02$ & $0.66 \pm 0.01$ & $99.74 \pm 0.01$ & $99.96 \pm 0.02$ & $99.85 \pm 0.01$ \\
P8 & MSCBM & $0.43 \pm 0.03$ & $0.14 \pm 0.02$ & $99.73 \pm 0.02$ & $99.90 \pm 0.04$ & $99.74 \pm 0.01$ \\
& a-MEM & $0.58 \pm 0.03$ & $0.21 \pm 0.01$ & $99.85 \pm 0.01$ & $99.83 \pm 0.01$ & $99.87 \pm 0.02$ \\
\cline { 2 - 7 } & IMDM & $0.23 \pm 0.06$ & $0.19 \pm 0.04$ & $99.89 \pm 0.02$ & $99.86 \pm 0.04$ & $99.89 \pm 0.04$ \\
\cline { 2 - 7 } & MSCBM & $0.49 \pm 0.04$ & $0.56 \pm 0.03$ & $99.68 \pm 0.05$ & $99.99 \pm 0.04$ & $99.69 \pm 0.03$ \\
\hline
\end{tabular}

\section{Discussion}

MSCs are more and more used in clinical application and research,and has become a research hotspot. Therefore, there is a great demand for large-scale MSCs cultures. The cell culture methods used in various laboratories have also changed greatly. Many researchers have conducted experimental studies on the expansion of MSCs by changing the culture conditions and medium components. For example, the functions of MSCs under hypoxic culture conditions have been studied [17]. Simulated microgravity (SMG) can promote the differentiation of MSCs [18]. Some researchers have expanded MSCs by Simulate the microenvironment of stem cells in vivo [19]. These methods have resulted in higher quantity, high quality and high activity MSCs. MSC complete culture medium is composed of basal medium plus supplements. FBS is recognized as providing some essential cell growth nutrients and was the most commonly used MSC culture supplement. However, FBS is derived from cattle, thus containing xenogeneic animal proteins. There are also risks of infectious viruses such as mad cow disease, and other potential animal-derived diseases. The nutrient compositions of the serum-free culture systems are balanced and controllable, which can avoid some hidden dangers caused by xenogeneic animal-derived proteins, so it has been increasingly recognized and applied by researchers. However, it has not been successful in culturing MSC in serum-free medium with completely known components. Nowadays, the most commonly used MSC culture supplement is platelet lysate. Some companies provide platelet lysate products with added growth factors as MSC culture supplements. This type of culture systems is superior 
to using FBS in many ways as discussed. Therefore, it has been widely accepted. Cells cultured in this way also do not cause immunogenic reactions induced by xenogeneic sera to the body in the treatment of human diseases.

On the other hand, there are many types of basal media for MSCs growth, and they differ in components and content amounts. Different basal media therefore have very different effects on cell growth, proliferation and differentiation potential. This issue has not seemed to get enough research. In a recent study, aMEM and DMEM as basal media supplemented with $10 \%$ of human platelet lysate (hPL) and DMEM supplemented with $20 \%$ fetal bovine serum (FBS) and bFGF were compared in culturing adipose tissue-derived mesenchymal stromal cells (ASCs). The results indicate thataMEM supplemented with $10 \%$ hPL yield the highest proliferation rate and rate of clonal genic potential, while no differences in adipocyte, osteocyte, and chondroblast differentiation[20]. This study was done with ASCs. As reported in the literature and in our own study, MSCs from different sources respond differently to cell culture basal media[In this study, the three commonly used low-cost basal media: MSCBM, a-MEM, and IMDM were pared with commercial platelet lysate product UltraGROGAdvanced. It is designed to select a costeffective and low-cost basal medium to use in serum-free culture system for large-scale production of human umbilical cord MSCs.

Our results of comprehensive analysis of cell proliferation indicators include the number of cell expansions in the same culture cycle, cell doubling time, growth curve measurement and the clonal analysis, showing that the cell proliferation functions of the three basal media are arranged from high to low as MSCBM, a-MEM, IMDM. Among them, many indexes of MSCBM and a-MEM are very close, while that of IMDM is obviously worse than the other two media. The cells cultured in the three media all have strong differentiation functions, and can all differentiate into osteoblasts, adipocytes, and chondrocytes. It is worth noting that the number of MSCBM-cultured cells differentiated into adipocytes was higher than that of IMDM-cultured cells $(0.01<\mathrm{P}<0.05)$, but there was no significant difference from a-MEM-cultured cells. The cells cultured in the three media were equally highly expressed MSCs specific markers CD73, CD90, CD105, and negatively expressed CD34, CD45. The percentage differences in the expression levels were not significant.

All three kinds of basal media are widely used. Although MSCBM is mainly distributed in China, its manufacture is actively expanding this product in other major markets. Based on the limited scope of the study,we also did not try to analyze the effects of individual components in the basal media.

\section{Conclusion}

In summary, based on our comprehensive analysis of cell proliferation indicators, also considering the cell morphology observation and differentiation potential analysis, we suggest the use of MSCBM or aMEM supplemented with 5\% UltraGROGAdvanced over the use of IMDM for cultivation of hUC-MSCs, especially for large-scale clinical applications. Our results should offer a good guidance on the selection of basal media for MSC cultures. 


\section{Declarations}

\section{Ethics approval and consent to participate}

This study protocol and informed consent form were approved by the Ethics Committee of the Tian Qing Stem Cell Co., Ltd. Harbin 150028, China. All human tissue donors signed the informed consent form to participate in this study and allow the publication of the results.

\section{Consent for publication}

Not applicable.

\section{Availability of data and materials}

The datasets during and/or analyzed during the current study available from the corresponding author on reasonable request.

\section{Competing interests}

The authors declare that they have no competing interests. This study did not receive any funding from any of the medium supplier.

\section{Funding}

This study was supported by internal funding from the Tian Qing Stem Cell Co., Ltd. The funding body takes no role in the design of the study and collection, analysis, and interpretation of data and in writing the manuscript.

\section{Authors' contributions}

L.W. designed and did most of the experimental work, analysis of data and initial writing of the manuscript in Chinese. C.L. performed part of the experimental work in tissue processing and cell cultures. H.L. carried out the adipogenic differentiation and clonal analysis. J.Y. was responsible for the rewriting of Chinese and English final versions, and the translation into English and the submission process. Y.Z. contributed to the study design and critical discussion.

All authors read and approved the final manuscript.

Corresponding authors: All correspondence to Yi Zhang.

\section{Acknowledgements}


The authors are grateful to all people who donated their umbilical cord tissue for this study. We sincerely acknowledge the staffs at the Production Department of the Tian Qing Stem Cell Co., Ltd. for their technical assistance in this study.

\section{References}

1. JiaoG, LouG, MoY, et al. Acombination of GDNF and hUCMSC transplantation loaded on SF/AGs composite scaffolds for spinal cord injury repair [J].Mater Sci Eng C Mater BiolAppl, 2017, 74:230237.

2. XiaoB, RaoF, GuoZY, etal.Extracellular matrix from human Umbilical cord-derived mesenchymal stem cells as a scaffold for Peripheral nervere generation [J].Neural Regen Res ,2016,11(7):1172-1179.

3. WuGY, QuanKY, LiM, etal.Humanum biblical cord derived schwann like cell transplantation combined with neurotrophin-i $1 \%$ " Administration in dyskinesia of rats with spinal cord injury [J].NeurochemRes ,2011,36(5):783-792.

4. YeB, LuoX, LiZ, etal.Rapid biomimetic mineralization of collagen fibrils and combining with humanum biblical cord mesenchymal stem cells for bone defects healing[J].MaterSciEngC MaterBiolAppl ,2016,68:43-51.

5. YanY, JiangW, TanY, etal.hucMSC Exosome-Derived GPXi $14^{\prime}$ Is Required for the Recovery of Hepatic Oxidant Injury[J].Mol Ther, 2017, 25(2):465-479.

6. BiZM, ZhouQF, GengY, etal.Humanum biblical cord mesenchymal stem cells ameliorate experimental cirrhosis through activation of keratinocyte growth factor by suppressing microR- NAi $1 / 4$ §-199[J].Eur Rev Med Pharmacol Sci,2016,20(23): 4905-4912.

7. Burnouf T, Strunk D, Koh MBC, Schallmoser K. Human platelet lysate: Replacing fetal bovine serum as a gold standard for human cell propagation? Biomaterials 2016:76:371-87.

8. Dozza B, Di Bella C, Lucarelli E, et al. Mesenchymal stem cells and platelet lysate in fibrin or collagen scaffold promote non-cemented hip prosthesis integration. Journal of orthopaedic research: official publication of the Orthopaedic Research Society 2011,29(6):961-968.

9. Lange C, Brunswig-Spickenheier B, Eissing L, Scheja L. Platelet lysate suppresses the expression of lipocalin-type prostaglandin D2 synthase that positively controls adipogenic differentiation of human mesenchymal stromal cells.Experimental cell research 2012,318 (18):2284-2296.

10. Abdelrazik H, Spaggiari GM, Chiossone L, Moretta L. Mesenchymal stem cells expanded in human platelet lysate display a decreased inhibitory capacity on T- and NK-cell proliferation and function. European journal of immunology 2011:41(11):3281-3290.

11. Perez-Ilzarbe M, Diez-Campelo M, Aranda P, et al. Comparison of ex vivo expansion culture conditions of mesenchymal stem cells for human cell therapy. Transfusion 2009:49(9):1901-1910.

12. von Bonin M, Stolzel F, Goedecke A, et al. Treatment of refractory acute GVHD with third-party MSC expanded in platelet lysate containing medium. Bone marrow transplantation 2009: 43(3):245-251. 
13. Trento C, Bernardo ME, Nagler A, et al.Manufacturing Mesenchymal Stromal Cells for the Treatment of Graft-versus-Host Disease: A Survey among Centers Affiliated with the European Society for Blood and Marrow Transplantation. Biol Blood Marrow Transplant,2018:24 (11): 2365-70.

14. Kinzebach S, Dietz L, Kluter $\mathrm{H}$, et al. Functional and differential proteomic analyses to identify platelet derived factors affecting ex vivo expansion of mesenchymal stromal cells. BMC cell biology 2013,14:48.

15. Menard C, Pacelli L, Bassi G, et al. Clinical Grade Mesenchymal Stromal Cells Produced Under Various Good Manufacturing Practice Processes Differ in Their Immunomodulatory Properties:Standardization of Immune Quality Controls. Stem cells and development 2013,22(12):1789-8001.

16. LiX, HuYD, ChenY, et al.Mechanisms of Improvement of Left Ventricular Function by Intracoronary Human Umbilica Cordi $1 / 4 \oint$ Derived Mesenchymal Stem Cell Infusionin Very Old Patients With Coronary Chronic Total Occlusion [J].Curr Pharm Des,2015,21(26):3844-3850.

17. NekantiU, RaoVB, BahirvaniAG, et al.Longī $1 / 4 \xi$ termexpansion and pluripotent marker array analysis of

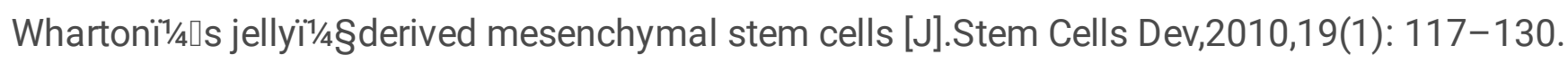

18. XueL, LiY, ChenJ, etal.Duration of simulated microgravity affects the differentiation of mesenchymal stem cells [J].Mol MedRep ,2017, 15(15): 3011-3018.

19. SCHung.Effects of hypoxic culture on bone marrow mesenchymal stem cells: from benchtobedside [J].Form osan JSurg,2013,46(2):35-38.

20. Czapla J, Matuszczak S, Kulik K, et al. The effect of culture media on large-scale expansion and characteristic of adipose tissue-derived mesenchymal stromal cells[J]. Stem Cell Research \& Therapy, 2019, 10(1).

\section{Figures}


Fig. 1
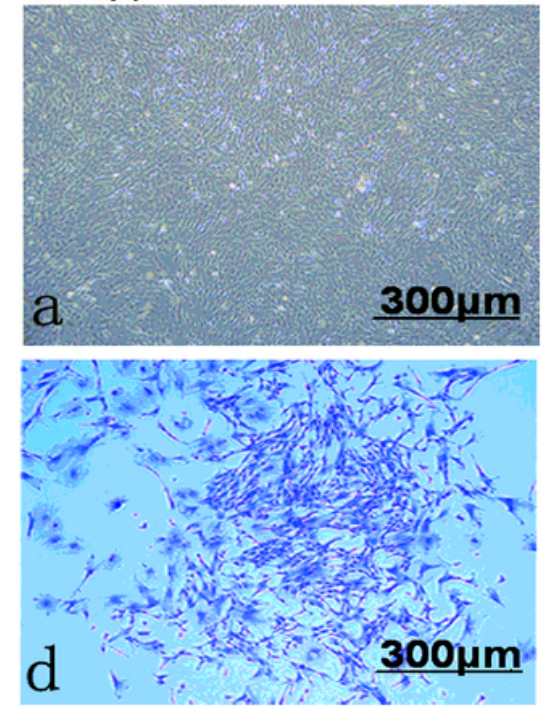

Cell proliferation function of $\mathrm{P} 3$
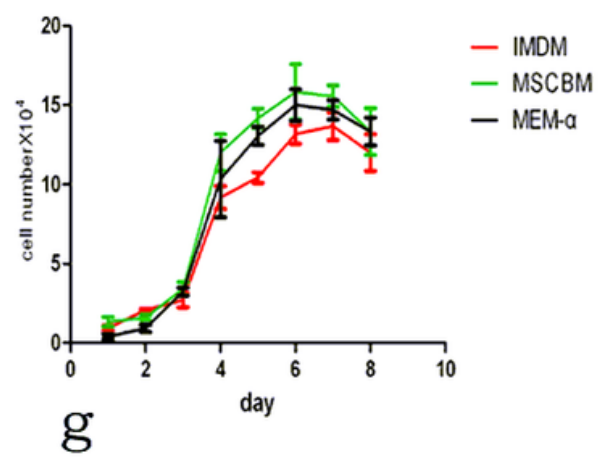
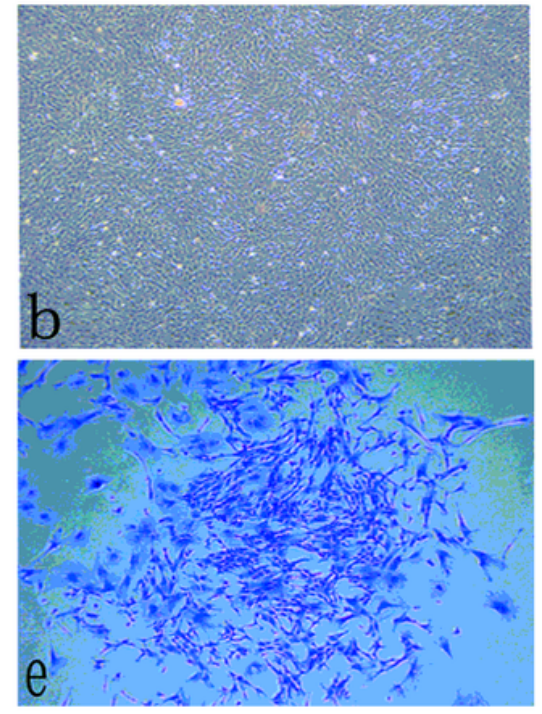

Cell proliferation function of P5

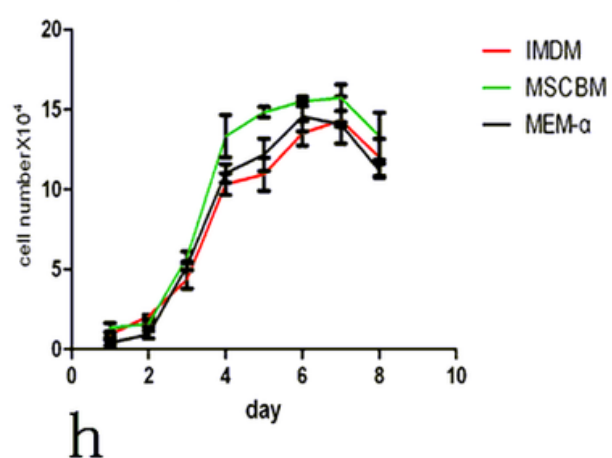

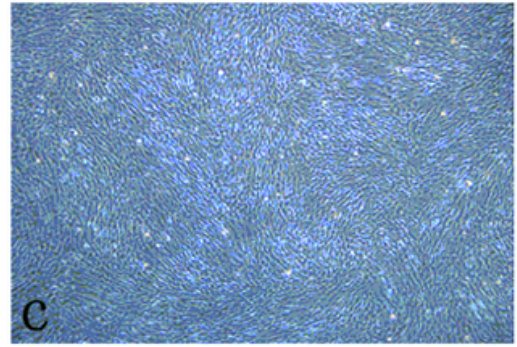

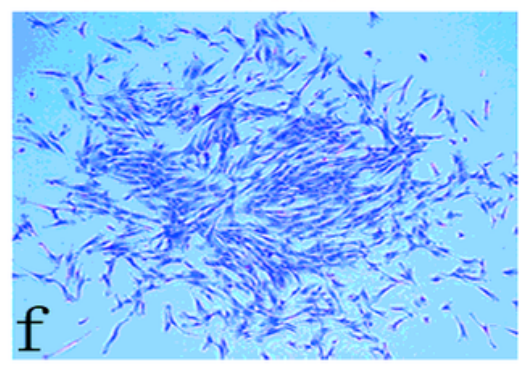

Cell proifferation function of $P 8$

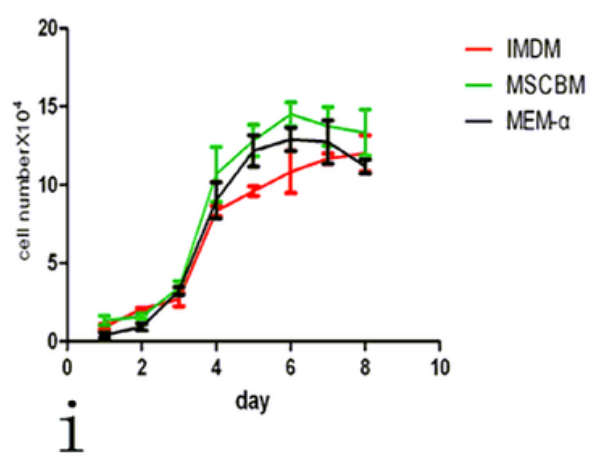

Figure 1

The morphological observations and growth curves. 
Fig. 2
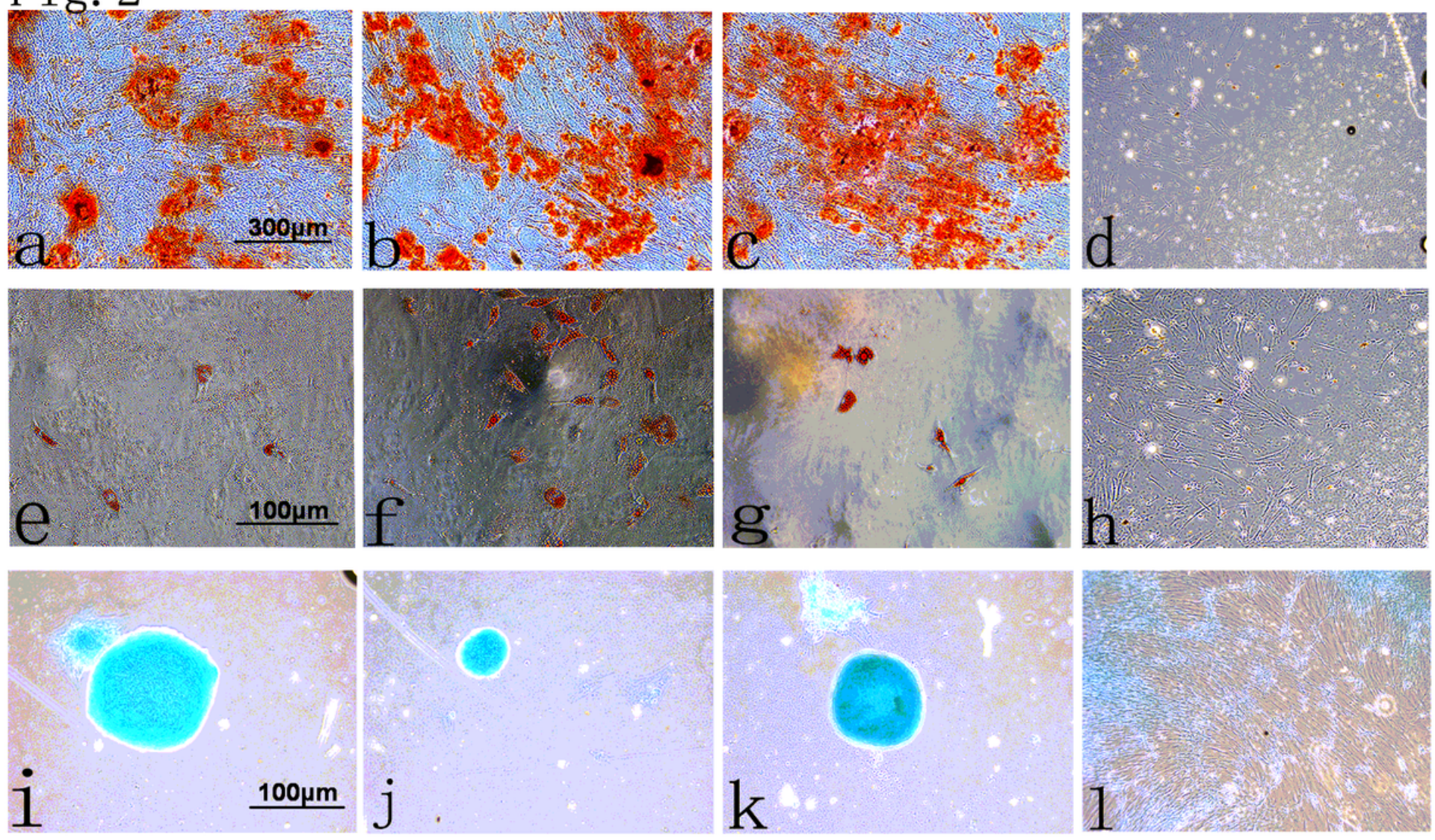

Figure 2

Osteogenic, adipogenic and chondrogenic differentiation by P8 hUC-MSCs. 